\title{
Black Phases of CsPbl3: Structural and Theoretical Studies
}

\author{
Laurent Pedesseau ${ }^{a}$, Arthur Marronnier ${ }^{b}$, Guido Roma ${ }^{c}$, Soline Boyer-Richard ${ }^{a}$, Jean-Marc \\ Jancu $^{a}$, Yvan Bonnassieux ${ }^{b}$, Claudine Katan ${ }^{d}$, Constantinos Stoumpos ${ }^{e}$, Mercouri Kanatzidis \\ e, Jacky Even ${ }^{a}$ \\ a, Univ Rennes, INSA Rennes, CNRS, Institut FOTON - UMR 6082, F-35000 Rennes, France \\ b, LPICM, CNRS, Ecole Polytechnique, Université Paris-Saclay, F-91128 Palaiseau, France \\ c, Service de Physique de l'Etat Condensé CEA, CNRS, Universite Paris Saclay CEA Saclay , l'orme des \\ merisiers bat 772, 91191 Gif sur Yvette Cedex FRANCE \\ d, Univ Rennes, ENSCR, INSA Rennes, CNRS, ISCR (Institut des Sciences Chimiques de \\ Rennes) - UMR 6226, F-35000 Rennes, France \\ e, Department of Chemistry and Argonne-Northwestern Solar Energy Research (ANSER) Center, \\ Northwestern University, Evanston, Illinois 60208, USA
}

In less than 10 years, hybrid organic-inorganic perovskites have emerged as a new generation of absorber materials for high-efficiency and low-cost solar cells [1], [2]. More recently, fully inorganic perovskite quantum dots (QD) also led to promising efficiencies [3], [4] and then become a serious alternative to hybrid organic-inorganic perovskites. Currently, the record efficiency for QD solar cells is obtained with $\mathrm{CsPbl}_{3}$. High resolution in-situ synchrotron XRD measurements have been performed on $\mathrm{CsPbl}_{3}$ as a function of the temperature and revealed a highly anisotropic variation of the lattice parameters. Moreover, $\mathrm{CsPbl}_{3}$ can be temporarily maintained in a perovskite-like structure down to room temperature, stabilizing a metastable perovskite polytype (black-phase) crucial for photovoltaic applications. Structural, vibrational and electronic properties of the three experimentally observed black phases are further scrutinized using theoretical approaches [5], [6]. A symmetry-based tight-binding model, calibrated with self-consistent GW calculations including spin-orbit coupling, affords further insight into their electronic properties. A Rashba effect is thus predicted for both cubic and tetragonal phases when using the symmetry breaking structures obtained through frozen phonon calculations.

[1] A. Kojima, et al., Organometal Halide Perovskites as Visible-Light Sensitizers for Photovoltaic Cells. J. Am. Chem. Soc. 2009, 131, 6050-6051.

[2] Best research-cell efficiencies; https://www.nrel.gov/pv/assets/images/efficiency-chart.png (accessed Nov 7, 2017).

[3] H. Bian et al., Graded Bandgap CsPbI2+xBr1-x Perovskite Solar Cells with a Stabilized Efficiency of 14.4\%, Joule (2018), https://doi.org/10.1016/j.joule.2018.04.012

[4] E. M. Sanehira, et al., Enhanced Mobility CsPbI3 Quantum Dot Arrays for Record-efficiency, High-voltage Photovoltaic Cells. Sci. Adv. 2017, 3, eaao4204.

[5] A. Marronnier et al., Structural Instabilities Related to Highly Anharmonic Phonons in Halide Perovskites. . J. Phys. Chem. Lett. 2017, 8, 2659-2665

[6] A. Marronnier et al., Anharmonicity and Disorder in the Black Phases of Cesium Lead lodide Used for Stable Inorganic Perovskite Solar Cells. ACS Nano 2018, 12, 3477-3486

This project has received funding from the European Union's Horizon 2020 program through a FET Open research and innovation action under the grant agreement No 687008. The information and views set out in this report are those of the author(s) and do not necessarily reflect the official opinion of the European Union. Neither the European Union institutions and bodies nor any person acting on their behalf may be held responsible for the use which may be made of the information contained herein. The ab initio simulations have been performed on HPC resources of CINES under the allocation 2017-[x2017096724] made by GENCI (Grand Equipement National de Calcul Intensif). 